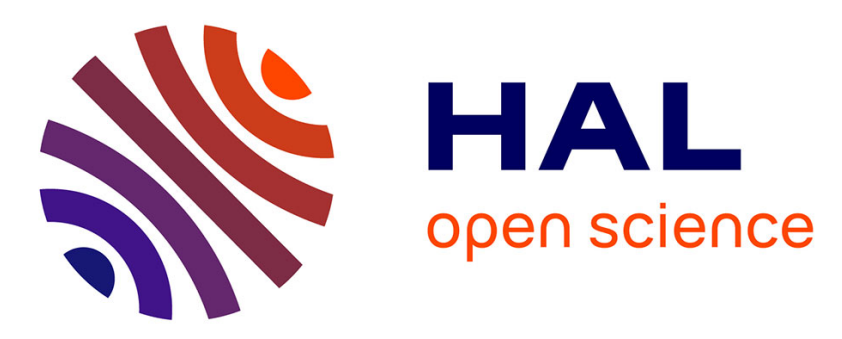

\title{
Ultimate Opening Combined with Area Stability Applied to Urban Scenes
}

Beatriz Marcotegui, Andrés Serna, Jorge Hernández

\section{To cite this version:}

Beatriz Marcotegui, Andrés Serna, Jorge Hernández. Ultimate Opening Combined with Area Stability Applied to Urban Scenes. 13th International Symposium on Mathematical Morphology and Its Applications to Signal and Image Processing, May 2017, Fontainebleau, France. pp.261-268, 10.1007/9783-319-57240-6_21. hal-01527983

\section{HAL Id: hal-01527983 \\ https: / hal-mines-paristech.archives-ouvertes.fr/hal-01527983}

Submitted on 26 May 2017

HAL is a multi-disciplinary open access archive for the deposit and dissemination of scientific research documents, whether they are published or not. The documents may come from teaching and research institutions in France or abroad, or from public or private research centers.
L'archive ouverte pluridisciplinaire HAL, est destinée au dépôt et à la diffusion de documents scientifiques de niveau recherche, publiés ou non, émanant des établissements d'enseignement et de recherche français ou étrangers, des laboratoires publics ou privés. 


\title{
Ultimate opening combined with area stability applied to urban scenes
}

\author{
Beatriz Marcotegui ${ }^{1}$, Andrés Serna ${ }^{2}$, and Jorge Hernández ${ }^{3}$ \\ 1 beatriz.marcotegui@mines-paristech.fr \\ MINES ParisTech, PSL Research University, \\ CMM - Centre for Mathematical Morphology \\ 35 rue Saint Honoré - Fontainebleau, France \\ http://cmm.mines-paristech.fr \\ 2 andres_serna@trimble.com \\ Trimble Inc., 3D Scanning Operating Site \\ 174 Av. du Maréchal de Lattre de Tassigny - Fontenay-Sous-Bois, France \\ http://www.trimble.com/3d-laser-scanning/ \\ 3 jorge.hernandez-londono@safrangroup.com \\ SAFRAN Paris-Saclay. Rue Des Jeunes Bois - Châteaufort, France
}

\begin{abstract}
This paper explores the use of ultimate opening in urban analysis context. It demonstrates the efficiency of this approach for street level elevation images, derived from 3D point clouds acquired by terrestrial mobile mapping systems. An area-stability term is introduced in the residual definition, reducing the over-segmentation of the vegetation while preserving small significant regions.

We compare two possible combinations of the Ultimate Opening and the Area Stability: first as a multiplicative factor, then as a subtractive term. On the one hand, multiplicative factor is very strict and many significant regions may be eliminated by the operator. On the other hand, a subtractive factor is more easily controlled according to the image dynamics. In our application, the latter provides the best results by preserving small meaningful objects such as poles and bollards while avoiding over-segmentation on more complex objects such as cars and trees.
\end{abstract}

Keywords: residual approach, ultimate opening, urban scene analysis, elevation images.

\section{Introduction}

Ultimate opening (UO) is a morphological operator based on numerical residues. It produces relevant partial partitions of generic images. The operator successively applies a decreasing family of openings, $\gamma_{\lambda}, \lambda=0,1, \ldots N-1$, that forms a size granulometry:

$$
\forall i, j: 0 \leq i \leq j \leq N-1 \Rightarrow \gamma_{i} \geq \gamma_{j}
$$

Then, the residues between successive openings are computed: $r_{i}=\gamma_{i}-\gamma_{i+1}$ and the maximum residue is kept for each pixel. Then, this operator returns two 
significant pieces of information for each pixel: $R(I)$, the maximal residue which carries contrast information and $q(I)$ which indicates the size of the opening leading to this residue, corresponding to the size of the structure containing the considered pixel. Thus, the ultimate opening definition is given by the following formula:

$$
\begin{aligned}
& R(I)=\max \left(r_{i}(I)\right)=\max \left(\gamma_{i}(I)-\gamma_{i+1}(I)\right), \forall i \geq 1 \\
& q(I)= \begin{cases}\max \left\{i+1 \mid r_{i}(I)=R(I)\right\} & \text { if } R(I)>0 \\
0 & \text { if } R(I)=0\end{cases}
\end{aligned}
$$

In this residual framework, other openings can be used: openings by reconstruction [15] or attribute openings [16]. These openings preserve the shape of the analyzed objects. Moreover attribute openings allow an efficient implementation based on a max-tree representation. Their use in real-time applications becomes possible for relatively large images.

Successful applications based on this operator have been developed such as rock analysis [13], automatic text localization [16], façade segmentation [9]. Morphological profiles are extensively used in the remote sensing field: they address classification approaches in urban areas $[2,3,10]$, ground and building extraction from lidar data $[14,12]$. As far as we know, these techniques have not been used for classification purposes on elevation images at the street level, acquired by terrestrial mobile mapping systems equipped with lidar scanners.

In this paper we apply ultimate opening to street level elevation images. We analyze the results and improve them introducing an area stability term in the residual definition. The paper is organized as follows: Section 2 details UO computation on a synthetic image. Section 3 introduces the area-stability term in the residual framework. Then, Section 4 demonstrates the efficiency of this method to segment urban scenes into significant objects. Finally, Section 5 concludes the paper and gives some perspectives for future developments.

\section{Ultimate opening computation}

Fig. 1 illustrates the intermediate steps of $U O$ calculation. The attribute opening [6] used in this example is the height (y-extent) of the bounding box containing a connected component. The associated function $q$ corresponds then to the height attribute of segmented structures. Fig. 1(a) shows the original image I. Results of successive height attribute openings (of size 1,2,3 and 4) are illustrated in the Fig. 1(a-d). Then residues are computed (Fig. 1(e-g)) and for each pixel, the maximum residue $r_{i}$ is selected and stored in $R(I)$ (Fig.1(h)) and the size of the opening leading to this residue is recorded in $q(I)$ (Fig.1(i)).

As aforementioned, the ultimate opening operator can be applied for segmenting generic images. In this paper, we explore its performance on elevation images from urban scenarios. Consider the Fig. 2, showing a 3D point cloud from Assas street in Paris. Fig. 2, first column, illustrates the ultimate height opening on the elevation image from that $3 \mathrm{D}$ point cloud. 

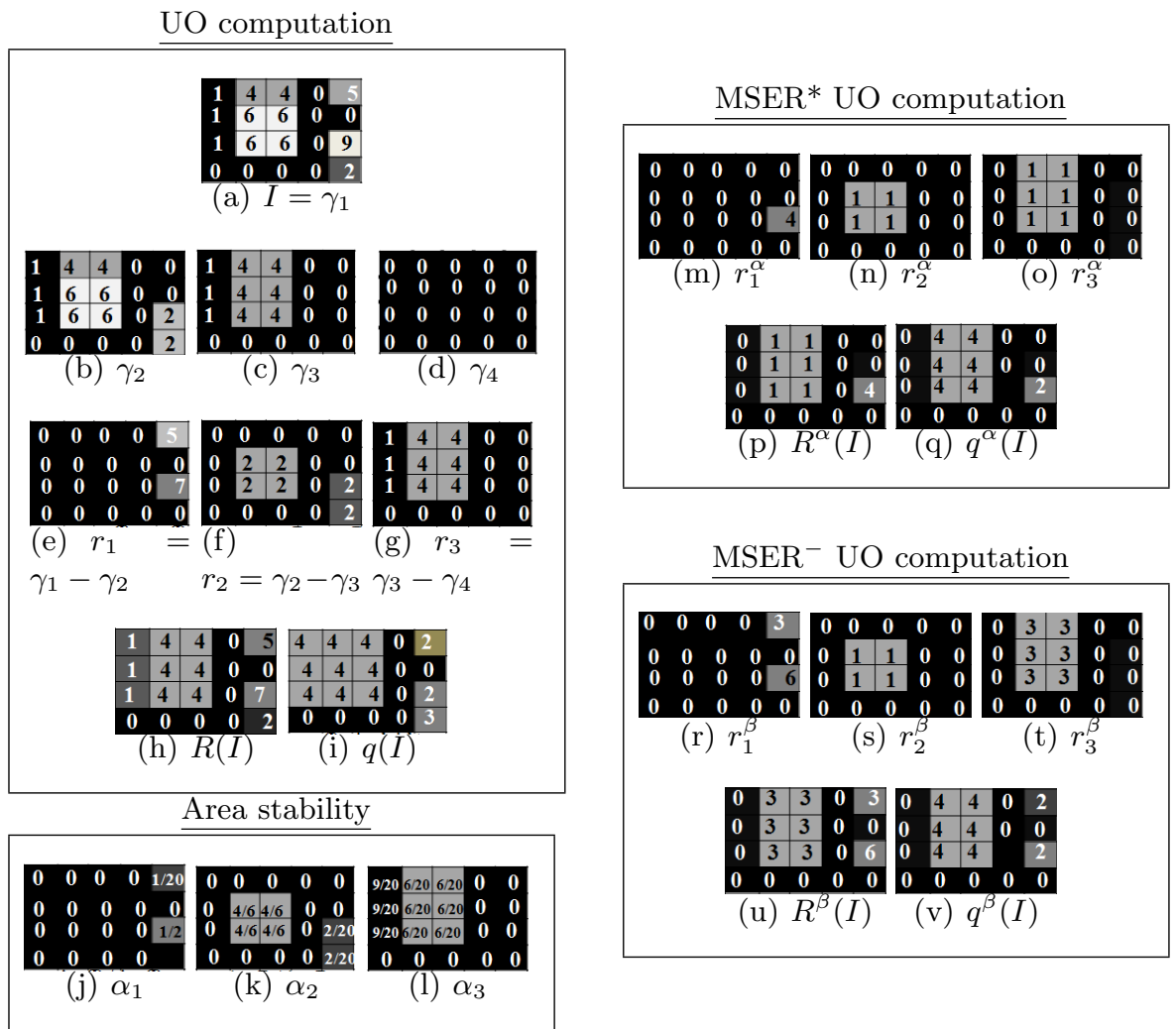

$\mathrm{MSER}^{-} \mathrm{UO}$ computation

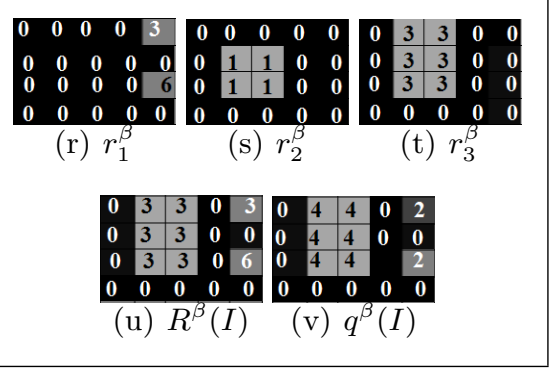

Fig. 1. Height $U O$ computation. (a) Input image $I$. (b-d) height openings. (e-g) residues. (h-i) resulting $R(I)$ and $q(I)$ images. (j-l) area stability. (m-o) MSER* residues and $(\mathrm{p}-\mathrm{q})$ resulting images. $(\mathrm{r}-\mathrm{t}) \mathrm{MSER}^{-}$residues and $(\mathrm{u}-\mathrm{v})$ resulting images.

\section{UO combined with area stability}

Despite its performance, a drawback of the ultimate opening is the numerous spurious regions that are segmented in the background. Belhedi et al. [1] address this problem. These spurious regions are assumed to correspond to noisy regions with low area stability (inspired from MSER approach [11]). In order to reduce the influence of noise [1] propose to introduce an area stability weighting factor, $\alpha_{i}$, in the residue definition:

$$
r_{i}^{\alpha}=r_{i} * \alpha_{i} \text { with } \alpha_{i}=\frac{\text { area }_{i}}{\text { area }_{i+1}}
$$

where area $_{i}$ is the area of the connected component of $r_{i}$ containing the considered pixel, in the threshold decomposition framework.

Fig. 1 illustrates the $M S E R^{*} U O$ computation on the previously used synthetic image. First, the area stability weighting factors, $\alpha_{i}$ are computed in 
Fig. 1(j-l). Then $r^{\alpha}$ are obtained multiplying $\alpha_{i}$ by $r_{i}$ in Fig. 1(m-o). Finally, the maximal residue is computed for each pixel in $R^{\alpha}$ and the corresponding attribute size in $q^{\alpha}$. Comparing Fig. 1(i) and Fig. 1(q), we can observe that $q^{\alpha}$ contains less noise than $q$

The result of $M S E R^{*} U O$ on an elevation image from a urban environment is shown in Fig. 2, second column. Many noisy regions have faded. However bollards dismiss too, as they are small regions that merge directly with large regions without transition zones. They are then considered as noisy regions and are filtered out.

The multiplicative factor has a high influence in the final residual value and as we have seen it may delete significant small regions. We propose to introduce the area stability information as a subtractive term instead of multiplicative factor. Thus, the residue is defined as:

$$
r_{i}^{\beta}=r_{i}-\beta_{i} \text { with } \beta_{i}=k *\left(1-\alpha_{i}\right)
$$

where $k$ is a maximal influence value, related to the image dynamics, given that the area stability is bounded between 0 and 1 . In that way, the influence of the stability can be controlled by factor $k$. Fig. 1(r-t) show the corresponding $M S E R^{-} U O$ residues and Fig. $1(\mathrm{u}-\mathrm{v})$ the resulting images. The region in the top right corner is preserved in spite of its low area stability.

Fig. 2, third column shows the result of the UO with a subtractive MSER term $\left(M S E R^{-} U O\right)$. In our experiments, the $k$ parameter has been chosen to be one hundredth of the maximum elevation of the image $(k=\max \{I\} / 100)$. Since the highest structures in the elevation image are trees and facades higher than 15 meters, this selected $k$ value allows to extract objects in the decimeter scale. We can observe that most noisy regions are not present anymore while preserving bollards and trees without over-segmentation. The third row shows a zoom of the second row in order to appreciate the differences between the three ultimate opening versions.

\section{Results}

A urban scene semantization contest was organized by the French Mapping Agency (IGN) [5]. 3D point clouds were acquired in Paris by Stereopolis, the IGN terrestrial mobile mapping system equipped with lidar sensors. The method leading to the best results on TerraMobilita/iQmulus database [21] apply to the elevation image obtained by a vertical projection of the $3 \mathrm{D}$ point cloud. This method, presented in $[20,18]$, is based on the hypothesis that each object contains only one significant maximum on the elevation image, which is true for objects such cars and poles. In that sense, the method relies on the $h$-maxima operator [17] to filter out low contrasted maxima and use the significant maxima as input for a constrained watershed [4] in order to obtain segmented objects. The main drawback of this method is that it does not work on complex objects such as vegetation and motorcycles. Moreover, the $h$ parameter is a key parameter: 
if it is too low it over-segments textured areas and if it is too high it removes significant regions.

Ultimate opening is a good candidate to deal with urban analysis on this type of images. Image interpolation based on the morphological fill-holes operator introduced in [8] and used also in $[20,18]$ is adopted in this paper in order to fill occluded areas and assure connectivity. The result of ultimate height opening applied to the interpolated image is shown in Fig. 2 first column. We can observe that the trees are correctly segmented, without over-segmentation and the car next to the trees is also correctly handled. Compared with the competing approach [19] the trees are not over-segmented and the car is correctly handled, even if it is not a maximum.

The maximal residue image $R$ produces many spurious regions in the background that do not correspond to regions of interest. A thresholding of $\mathrm{R}$ can help removing these regions but may also remove low contrasted regions. The use of area stability weight penalizes regions with important changes in area that are probably related to the presence of noise, artifacts and contrast variation in the background or unintended connection between components.

Fig. 3 and Fig. 4 show additional results on elevation images derived from 3D point clouds acquired by the CAOR-MINES ParisTech acquisition system [7]. The same remarks on the quality of the result are valid for these images.

\section{Conclusions and perspectives}

This paper demonstrates the interest of the ultimate opening for the analysis of urban scenes. It produces relevant partitions avoiding over-segmentation of trees as well as preserving the objects next to them, even if they do not correspond to regional maxima in the elevation images. A drawback of this operator is that it produces many noisy regions in the background. The combination of an area stability term in the residual definition highly reduces this problem. We propose to use a subtraction term instead of a multiplicative factor previously introduced in the literature. In our experiments, we show that this subtractive term better handles significant small regions while efficiently removing noisy ones. It amounts to applying an adaptive contrast threshold that depends on the area stability of the region. In future work, we will quantify the benefit of our approach in urban scene analysis.

\section{References}

1. A. Belhedi and B. Marcotegui. Adaptive scene-text binarisation on images captured by smartphones. IET Image Processing, 10(7):515-523, 2016.

2. J.A. Benediktsson, K. Arnason, and M. Pesaresi. The use of morphological profiles in classification of data from urban areas. In Remote Sensing and Data Fusion over Urban Areas, IEEE/ISPRS Joint Workshop 2001, pages 30-34. IEEE, 2001. 

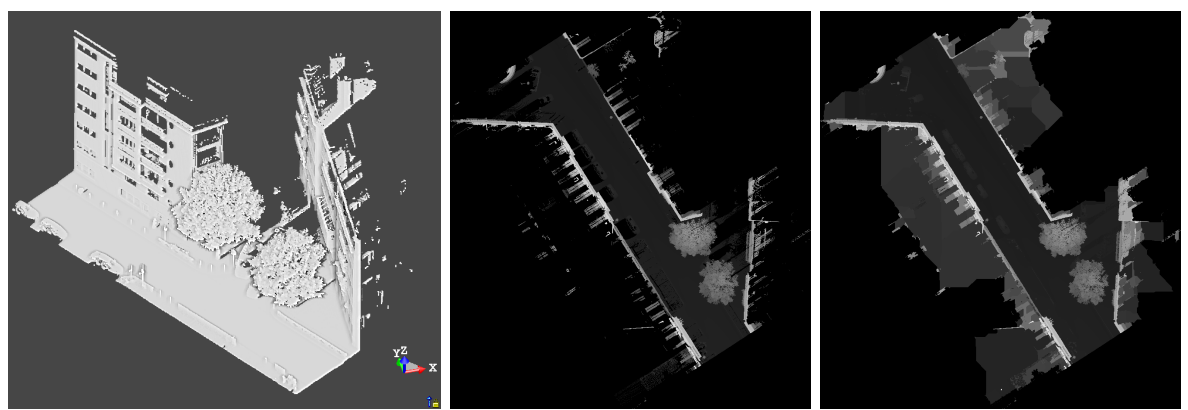

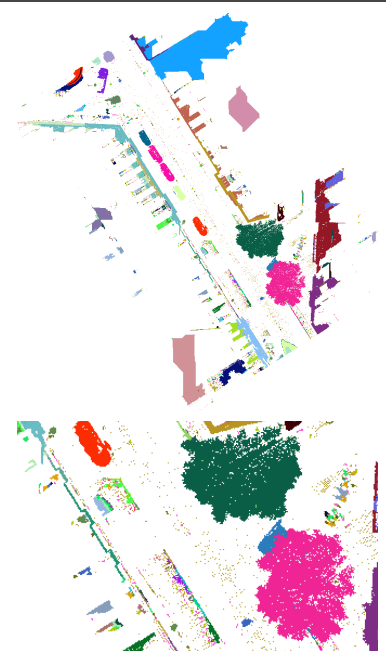

$\mathrm{UO}$

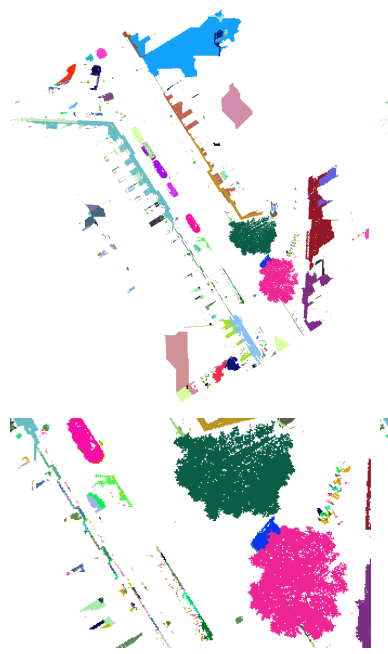

MSER*UO

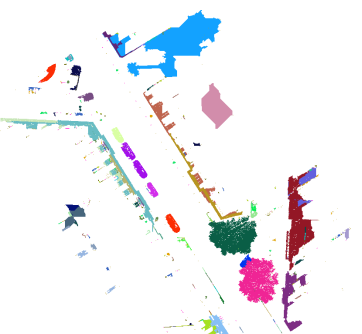

Fig. 2. Ultimate opening results on Assas street, Paris (TerMob2_LAMB93_0021 point cloud acquired by IGN). First row: 3D point cloud, elevation image $I$ and its interpolated version $\hat{I}$. Second row: $\mathrm{q}(\hat{I})$. Third row: a zoom on second row for visualization purposes.

3. J.A. Benediktsson, M. Pesaresi, and K. Arnason. Classification and feature extraction for remote sensing images from urban areas based on morphological transformations. IEEE Trans. Geoscience and Remote Sensing, 41(9):1940-1949, September 2003.

4. S. Beucher and C. Lantuéjoul. Use of watersheds in contour detection. In International workshop on image processing, real-time edge and motion detection, 1979.

5. M. Brédif, B. Vallet, A. Serna, B. Marcotegui, and N. Paparoditis. Terramobilita/iqmulus urban point cloud classification benchmark. In Workshop on Processing Large Geospatial Data, 2014.

6. E. J. Breen and R. Jones. Attribute openings, thinnings, and granulometries. Computer Vision and Image Understanding, 64(3):377-389, 1996.

7. F. Goulette, F. Nashashibi, S. Ammoun, and C. Laurgeau. An integrated onboard laser range sensing system for on-the-way city and road modelling. The 

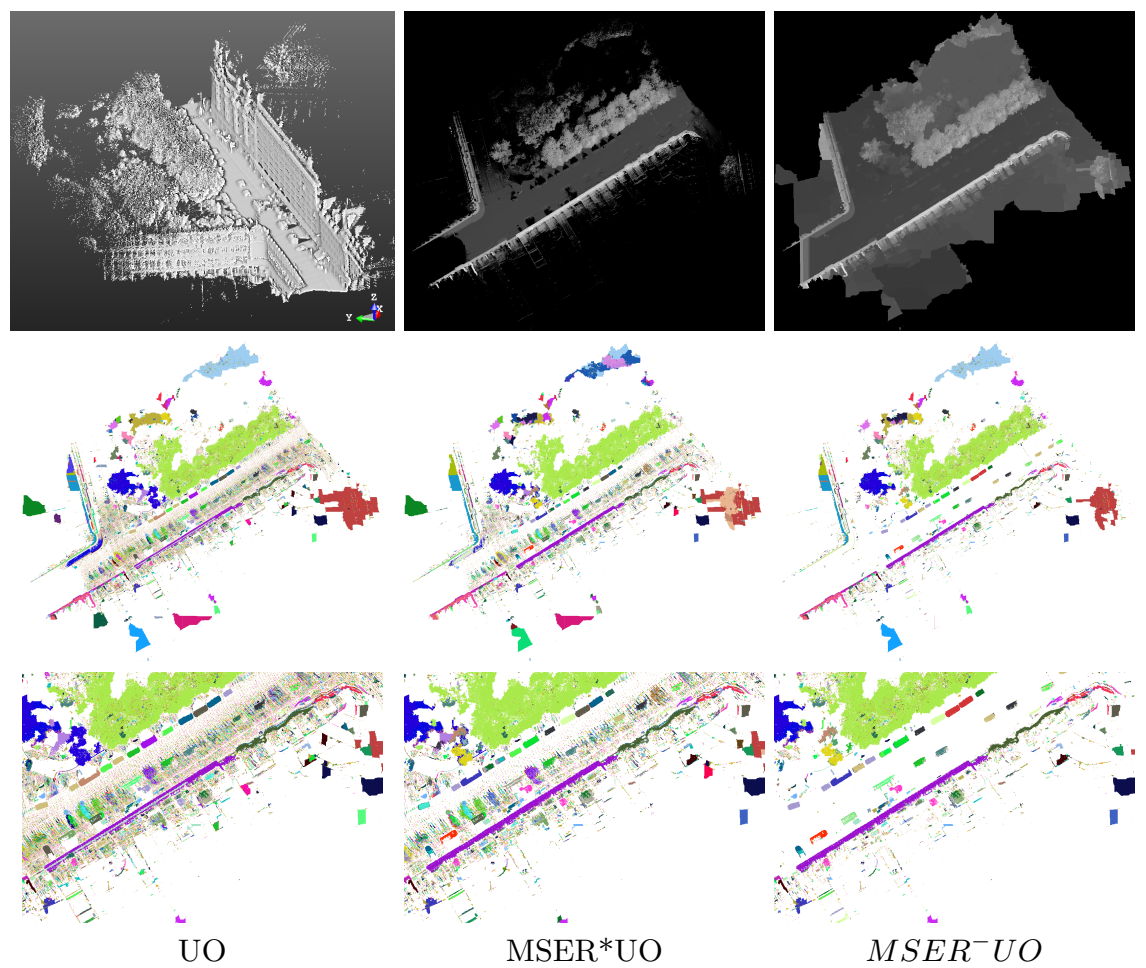

Fig. 3. Ultimate opening results on Montparnasse street, Paris (acquired by CAORMINES ParisTech). First row: 3D point cloud, elevation image $I$ and its interpolated version $\hat{I}$. Second row: $\mathrm{q}(\hat{I})$. Third row: a zoom on second row for visualization purposes.

ISPRS International Archives of Photogrammetry, Remote Sensing and Spatial Information Sciences, XXXVI-1:1-6, 2006.

8. J. Hernández and B. Marcotegui. Segmentation et interprétation de nuages de points pour la modélisation d'environnements urbains. Revue Francaise de Photogrammetrie et de Teledetection, (191):28, 2008.

9. J. Hernández and B. Marcotegui. Ultimate attribute opening segmentation with shape information. In Proceedings of the 9th International Symposium on Mathematical Morphology and Its Application to Signal and Image Processing, ISMM '09, pages 205-214, Berlin, Heidelberg, 2009. Springer-Verlag.

10. S. Lefevre, J. Weber, and D. Sheeren. Automatic building extraction in vhr images using advanced morphological operators. Proc. IEEE/ISPRS Joint Workshop Remote Sensing and Data Fusion over Urban Areas, 2007, 2007.

11. J. Matas, O. Chum, M. Urban, and T. Pajdla. Robust wide baseline stereo from maximally stable extremal regions. Proc. of British Machine Vision Conference, pages $384-396,2002$.

12. D. Mongus, N. Lukac, and B. Zalik. Ground and building extraction from LiDAR data based on differential morphological profiles and locally fitted surfaces. ISPRS Journal of Photogrammetry and Remote Sensing, 93:145-156, 2014. 


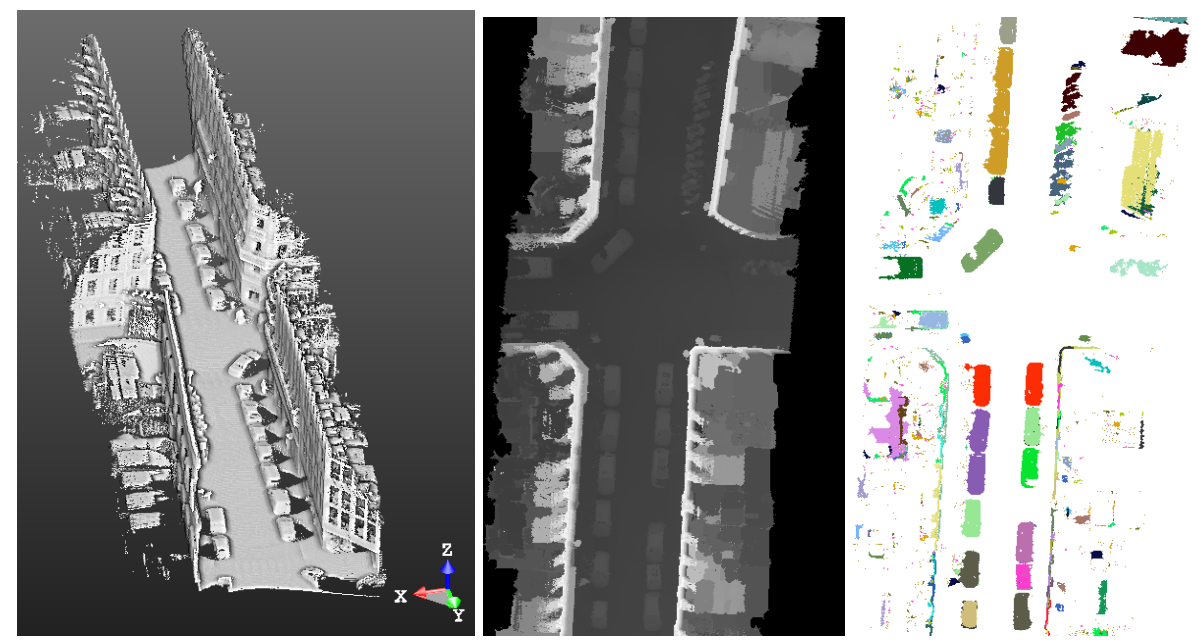

Fig. 4. Ultimate opening results on Madame street, Paris (point cloud acquired by CAOR MINES ParisTech). Left: 3D rendering. Middle: interpolated elevation image $\hat{I}$. Right: $\mathrm{q}(\hat{I})$.

13. S. Outal and S. Beucher. Controlling the ultimate openings residues for a robust delineation of fragmented rocks. In The 10th European Congress of Stereology and Image Analysis, June 2009.

14. M. Pedergnana, P.R. Marpu, M. Dalla Mura, J.A. Benediktsson, and L. Bruzzone. Classification of remote sensing optical and lidar data using extended attribute profiles. IEEE Journal on Selected Topics in Signal Processing, 6(7):856-865, 2012.

15. M. Pesaresi and J.A. Benediktsson. A new approach for the morphological segmentation of high-resolution satellite imagery. Geoscience and Remote Sensing, IEEE Transactions on, 39(2):309 -320, feb 2001.

16. T. Retornaz and B. Marcotegui. Scene Text Localization based on the Ultimate Opening. In Proc. of the 8th Intern. Symposium on Mathematical Morphology, volume 1 of ISMM 'O\%, pages 177-188, Rio de Janeiro, Brazil, October 2007.

17. M Schmitt and F Prêteux. Un nouvel algorithme en morphologie mathématique: les rh maxima et rh minima. Proc. 2ieme Semaine Internationale de l'Image Electronique, pages 469-475, 1986.

18. A. Serna. Semantic analysis of $3 D$ point clouds from urban environments: ground, facades, urban objects and accessibility. PhD thesis, Mines ParisTech - C.M.M., Fontainebleau - France, december 2014.

19. A. Serna and B. Marcotegui. Detection, segmentation and classification of $3 \mathrm{~d}$ urban objects using mathematical morphology and supervised learning. ISPRS Journal of Photogrammetry and Remote Sensing, 93(0):243 - 255, 2014.

20. A. Serna, B. Marcotegui, E. Decenciere, T. Baldeweck, A.-M. Pena, and S. Brizion. Segmentation of elongated objects using attribute profiles and area stability: Application to melanocyte segmentation in engineered skin. Pattern Recognition Letters, 47(0):172 - 182, 2014. Advances in Mathematical Morphology.

21. B. Vallet, M. Brédif, A. Serna, B. Marcotegui, and N.s Paparoditis. Terramobilita/iqmulus urban point cloud analysis benchmark. Computers \& Graphics, 49:126-133, 2015. 